Journal of Innovative Image Processing (JIIP) (2020)

Vol.02/ No. 02

Pages: 110-120

https://www.irojournals.com/iroiip/

DOI:_https://doi.org/10.36548/jiip.2020.2.006

\title{
Semi-Automated Segmentation Scheme for Computerized Axial Tomography Images of Esophageal Tumors
}

\author{
Dr. Bindhu V, \\ Professor, Head, \\ Department of Electronics and Communication Engineering, \\ PPG Institute of Technology, \\ Villankurichi Saravanampatti P.O, \\ Coimbatore, Tamil Nadu, India \\ Email Id: vbindhuppg@igmail.com
}

\begin{abstract}
The swift imaging speed, improved spatial resolution and lower cost compared to the magnetic resonance imaging is the reason behind the increased popularity of the computed axial tomography. In case of the esophageal tumor. The computed axial tomography utilizes the X-ray images to deliver the comprehensive images of the esophagus and the tissues surrounding it. It enables the detection of tumors or structural changes even at the developing stage and also gives the clear picture of the other distant organs like, lungs or liver affected by the cancer in the developed stage. One of the primary challenging chore in segmenting the esophageal tumor is because of the continuous variations in the position and the texture, intensity and as well as shape causing complexities in developing an standard procedure that could be applied universally. In this paper a semi-automated scheme is utilized in the segmentation of the esophageal tumors observed form the X-ray images of the computed axial tomography. An active- contour based semi-automated segmentation along with the procedures of level set is followed in the paper to segment the affected areas from the images of the esophagus acquired form the computed axial tomography. The strategy put forward segregates the work carried out into four major phases. The first phase extracts the images using the seed points. Second phase removes the unnecessary portions in the images, the threshold values are set in the third phase and the post processing is carried out in the fourth phase. This concept was evaluated on the real life data set of tumors collected from the nearby cancer treatment hospitals. The efficiency of the proposed strategy was compared with the previously existing state of art methods on the basis of the dice similarity, mean, medium and the maximal surface distance, and the Jaccard similarity. The concept put forth minimize the time utilization and also allows to have enhanced visualization of the tumors in the esophagus.
\end{abstract}

Keywords: Esophageal Tumor, Semi-Automated- Segmentation, Active -Contour, Computed Axial Tomography, Enhance Visualization

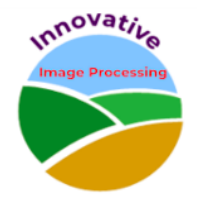


Journal of Innovative Image Processing (JIIP) (2020)

Vol.02/ No. 02

Pages: $110-120$

https://www.irojournals.com/iroiip/

DOI:_https://doi.org/10.36548/jiip.2020.2.006

\section{Introduction}

A comprehensive and appropriate diagnosis of cancer is the primary stage for the treatment of the esophageal cancer. Various tools and the tests are developed for the diagnosing the dangerous cancer causing tumors in the esophagus and evaluate the disease stage to develop the necessary "individualized treatment". The treatment is processed as several stages and in every stage the physician has to monitor the size of the tumors and develop the responsive treatment and vary the treatment plans whenever it is necessary to. Some of the regular procedures used in diagnosing are the "advanced genomic testing", upper endoscopy, biopsy, endoscopic ultrasound, Magnetic resonance imaging, and computed tomography or the computed axial tomography. Among the abovementioned tools in diagnosing the computed axial tomography is one of the highly and most extensively used tool as it allows have a swift imaging speed, improved spatial resolution and lower cost compared to the magnetic resonance imaging and the other tools. The computed axial tomography utilizes the X-ray images to deliver the comprehensive images of the esophagus and the tissues surrounding it. It enables the detection of tumors or structural changes even at the developing stage and also gives the clear picture of the other distant organs like, lungs or liver affected by the cancer in the developed stage. The tumors develop in the esophagus (a long hollow tune like organ that runs form the throat to the mouth) that assists in swallowing the food particles from the back of the throat to the stomach so that it gets digested. The tumors in the esophagus is the beginning phase of the cancer, it usually initiates in the cells that are lined in the interior of the esophagus. The tumors could occur in any location along the esophagus that is displayed in the figure. 1 below. The cancer has to be identified in the very early stage to have a better chance of recovery. In the later stages it can be treated but rarely cured, so the computed axial tomography which could the tumors or structural changes even at the developing stage is used for imaging the esophagus.

Yet few primary challenging chore exits in segmenting the esophageal tumor because of its continuous variations in the position and the texture, intensity and as well as shape causing complexities in developing an standard procedure that could be applied universally. So the paper utilizes a semi-automated scheme in the segmentation of the esophageal tumors observed form the X-ray images of the computed axial tomography. An active- contour based semi-automated segmentation along with the procedures of level set is followed in the paper to segment the affected areas from the images of the esophagus acquired form the computed axial tomography. The strategy put forward segregates the work carried out into four major phases. The first phase extracts the images using the seed points. Second phase removes the unnecessary portions in the images, the threshold values are set in the third phase and the post processing is carried out in the fourth phase.

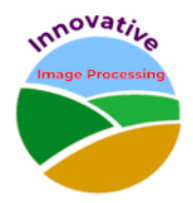


Journal of Innovative Image Processing (JIIP) (2020)

Vol.02/ No. 02

Pages: 110-120

https://www.irojournals.com/iroiip/

DOI:_https://doi.org/10.36548/jiip.2020.2.006

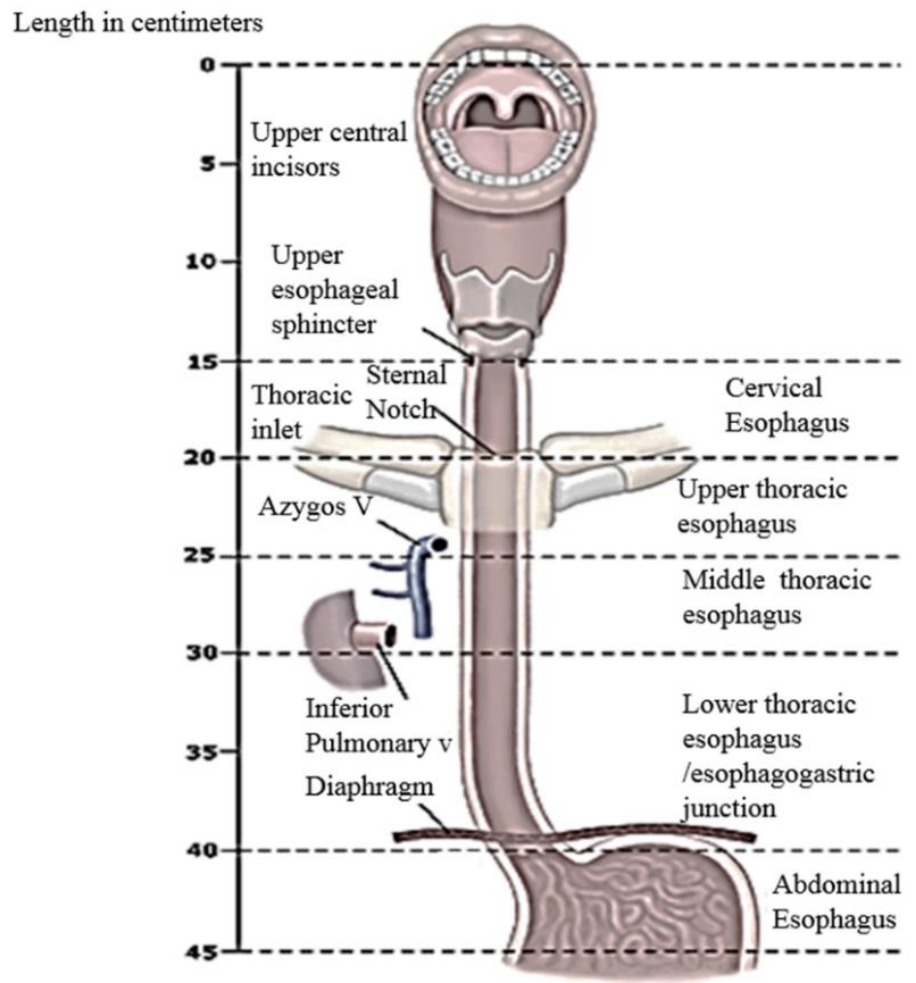

Figure.1 Structure of Esophagus [1]

This concept was evaluated on the real life data set of tumors collected from the nearby cancer treatment hospitals. The efficiency of the proposed strategy was compared with the previously existing stat of art methods on the basis of the dice similarity, mean, medium and the maximal surface distance, and the Jaccard similarity. The concept put forth minimize the time utilization and also allows to have enhanced visualization of the tumors in the esophagus

The remaining of the paper is arranged with the related studies presenting the previous works involved in the segmentation of the tumors that occurs anywhere in the human body in part 2, the proposed semiautomated contour-active segmentation for segmenting the esophageal tumors in part 3 , the performance evaluation on the basis of dice similarity, mean, medium and the maximal surface distance, and the Jaccard similarity in part 4 and conclusion in part 5.

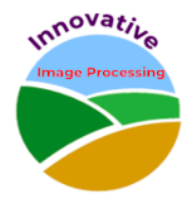


Journal of Innovative Image Processing (JIIP) (2020)

Vol.02/ No. 02

Pages: $110-120$

https://www.irojournals.com/iroiip/

DOI:_https://doi.org/10.36548/jiip.2020.2.006

\section{Related Studies}

The previous works based on the various automated and diverse procedures that were developed in segmenting the tumors affecting any parts of the body is discussed in this section. Gibson et al [2] utilized automated dense $\mathrm{v}$ networks to segment the multiple organ in the abdomen based on the images observed in the computed tomography. Carvalho, et al [3] presents a systematic review, on the three dimensional segmentation algorithms for the CT images. Koresh et al [4] has proposed a "Quantization with Perception for Performance Improvement in HEVC for HDR Content." Lenchik et al [5] presents a review on the automated tissue segmentation of the images acquired from the magnetic resonance imagining and computed tomography Tam, et al [6] discusses the "Machine Learning towards General Medical Image Segmentation."

Horie, et al [7] has conducted the "Quantitative Imaging Using Computed Tomography for Evaluation of Chronic Lung Allograft Dysfunction." Manoharan, Samuel et al [8] presents the "A Smart Image Processing Algorithm for Text Recognition Information Extraction and Vocalization for the Visually Challenged." Smith, et al [9] devised method of identifying the different stages of the liver fibrosis, Islam et al [10] proposed the region growing for the skin detection from the images and the videos. Bindhu, V. et al [11] conducted the "Biomedical Image Analysis using Semantic Segmentation."

Anshad, et al [12] utilized the modified region growing for segmenting the chondroblastoma Teng, et al [13] presented the "Improved krill group-based region growing algorithm for image segmentation." Anter, et al [14] has used the "hybrid approach using neutrosophic sets, fast fuzzy c-means and adaptive watershed algorithm" to segment the tumors in the CT images of the liver. Song et al [15] has conducted the "An Improved Confidence Connected Liver Segmentation Method Based on Three Views of CT Images." Sathesh, et al [16] devised the "Performance Analysis of Granular Computing Model in Soft Computing Paradigm for Monitoring of Fetal Echocardiography." Dias, et al [17] presented the "Semantic segmentation refinement by Monte Carlo region growing of high confidence detections." Nagesh, A et al [18] put forth the "An improved iterative watershed and morphological transformation techniques for segmentation of microarray images." Fourati, et al [19] presented the "Trabecular bone image segmentation using iterative watershed and multi resolution analysis." Masoumi, et al [20] presented an "Automatic liver segmentation in MRI images using an iterative watershed algorithm and artificial neural network." Yazdanpanah, et al [21] devised "Segmentation of intra-retinal layers from optical coherence tomography images using an active contour approach." Karami et al [22] "A semi-automated technique for internal jugular vein segmentation in ultrasound images using active contours." The proposed method in the paper utilizes the active- contour based semi-automated segmentation along with the procedures of level set to segment the tumors in the esophagus.

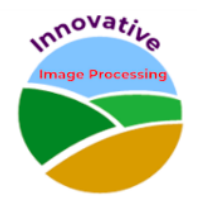


Journal of Innovative Image Processing (JIIP) (2020)

Vol.02/ No. 02

Pages: 110-120

https://www.irojournals.com/iroiip/

DOI:_https://doi.org/10.36548/jiip.2020.2.006

\section{Proposed Work}

The work proposed in the paper processes through different phases, the four important phases involved in the process is depicted in flowchart in figure. 2

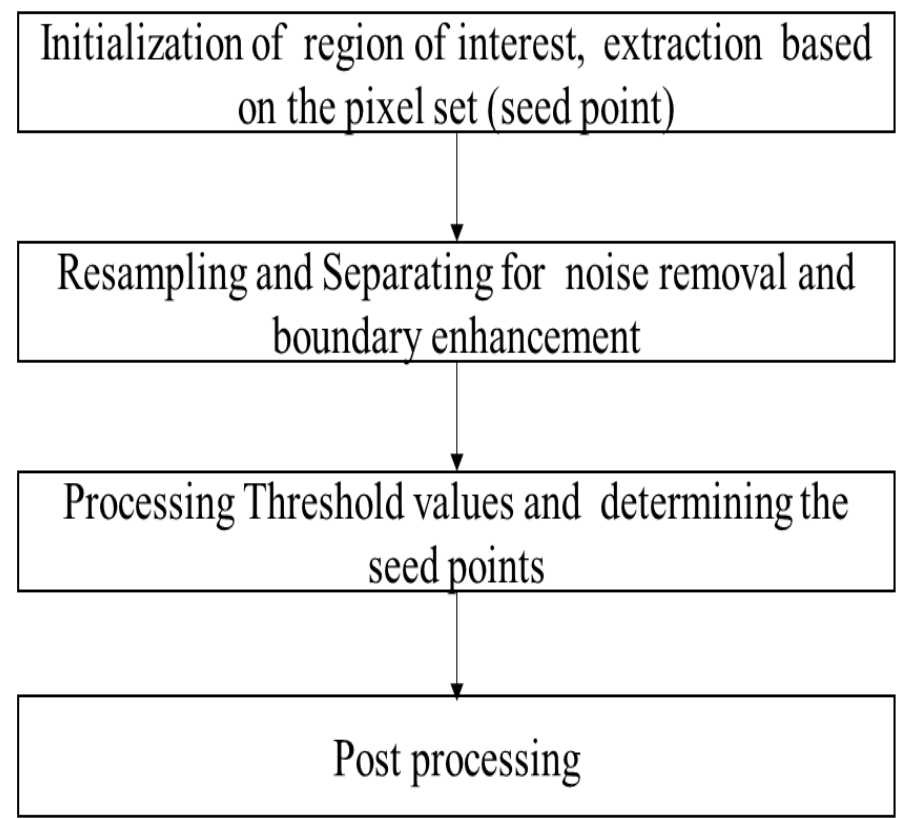

Figure.2 Flowchart of the Proposed Segmentation

The pixels (seed positions) in the images of the esophagus tumor is determined and using the pixels set the three dimensional region of interest for the esophagus tumor is estimated in the first phase, in the next phase the accurate parameters are initialized to resample the region of interest and minimize the noise signals in the image improving the structures of the tumors in the esophagus. The proposed method utilizes the anisotropic diffusion procedure to enhance the image structures, the unwanted noise signals present in the CT images would minimize the filter capacity in large regions growth. The "curvature diffusion procedure" eludes the noises in the images and protects the important structures such as the boundaries etc. this image that has been enhanced removing the noises is then subjected to boundary enhancement. The third phase adjust the minimum and the maximum threshold values and sets the beginning pixels to initiate the growth of the active -contours.

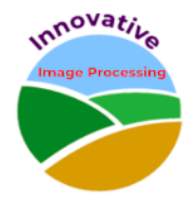


Journal of Innovative Image Processing (JIIP) (2020)

Vol.02/ No. 02

Pages: 110-120

https://www.irojournals.com/iroiip/

DOI:_https://doi.org/10.36548/jiip.2020.2.006

The values of the pixels that are between the minimum and the maximum threshold values are added into the segmentation region and other pixels that doesn't come under threshold values are excepted. The minimum and the maximum threshold values should be neither too close nor too apart to avoid the restriction on the "region on growth" or the inclusion of the entire image respectively. The output is received in the binary form with values assigned on the extracted regions and the other regions remaining zero.

The values of the intensity set is into the regions are extricated, and the pixels initially set is also permitted to grow in order to reach the boundaries. Once the outcome is observed in the binary form the final phase that does the post processing is done to deliver a three dimensional visualization. The figure. 3 presents the image acquired in the output of the every phase.
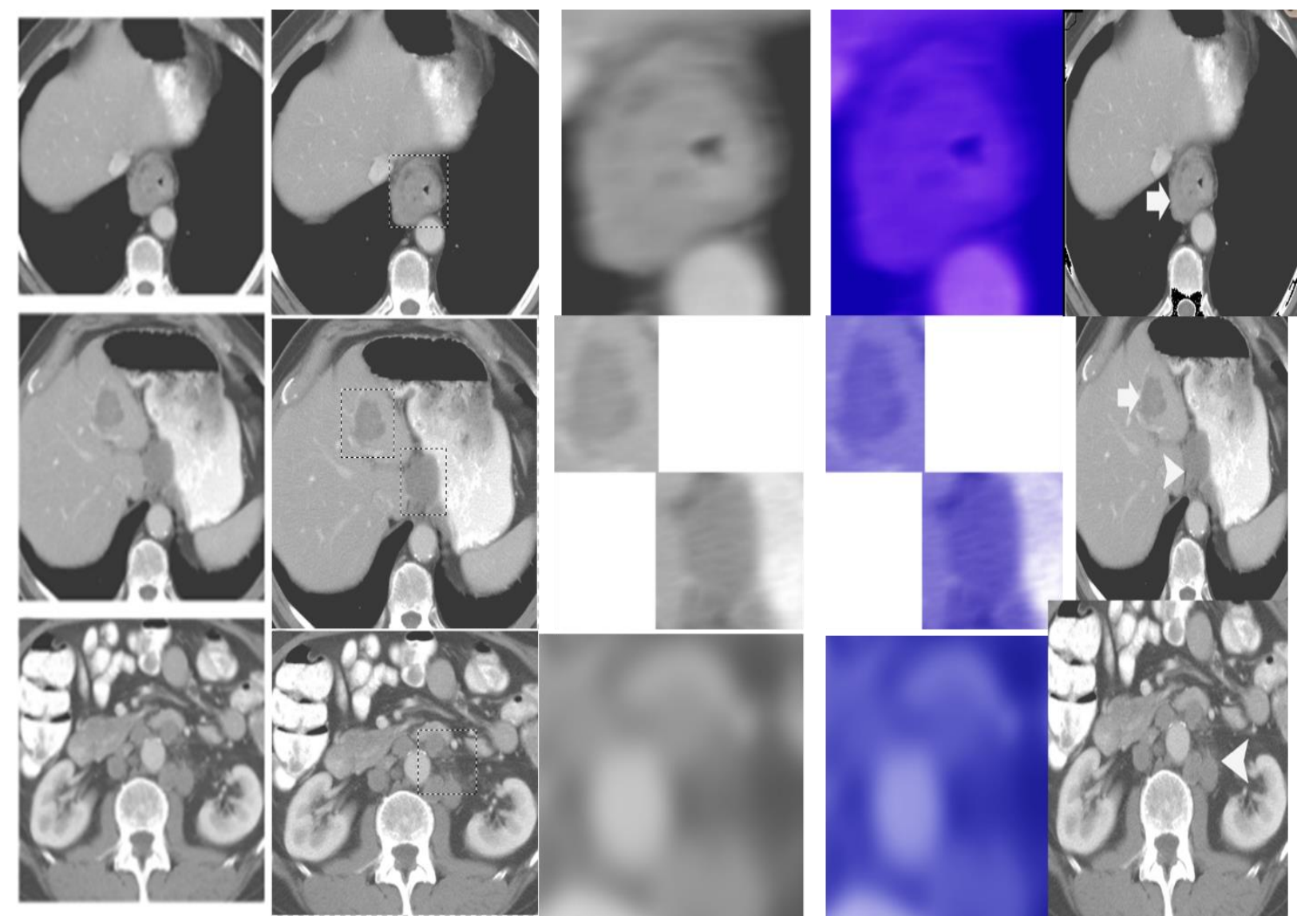
Journal of Innovative Image Processing (JIIP) (2020)

Vol.02/ No. 02

Pages: 110-120

https://www.irojournals.com/iroiip/

DOI:_https://doi.org/10.36548/jiip.2020.2.006

\section{Performance Analysis}

This concept was evaluated on the real life data set of tumors collected from the nearby cancer treatment hospitals. The efficiency of the proposed strategy was compared with the previously existing state of art methods on the basis of the dice similarity, mean, medium and the maximal surface distance, and the Jaccard similarity five different data sets based on the tumor occurrences in the different portions of the esophagus ("cervical esophagus, upper esophagus, middle thoracic esophagus, lower thoracic esophagus, abdominal esophagus") was gathered from the cancer treatment hospital. The data set collected had the information of twenty five patients with five patients on each type of cancer. Every set has about 100 images of the Computed Tomography on the tumors developed in the esophagus. All the images gathered were acquired form the records of the patients in the developing stage. The table. 1 below shows the details of the dataset.

\begin{tabular}{|l|l|l|l|}
\hline S.No & Dataset & Tumor occurred parts & No. of. Images \\
\hline 1 & Dataset 1 & Cervical Esophagus & 100 images \\
\hline 2 & Dataset 2 & $\begin{array}{l}\text { Upper thoracic } \\
\text { esophagus }\end{array}$ & 89 images \\
\hline 3 & Dataset 3 & $\begin{array}{l}\text { Middle thoracic } \\
\text { esophagus }\end{array}$ & 90 images \\
\hline 4 & Dataset 4 & $\begin{array}{l}\text { Lower thoracic } \\
\text { esophagus } \\
\text { esophagogastric junction }\end{array}$ & 97images \\
\hline 5 & Dataset 5 & Abdominal Esophagus & 95 images \\
\hline
\end{tabular}

Table.1 Data Set used

The performance metrics like dice similarity, mean, medium and the maximal surface distance, and the Jaccard similarity were used to evaluating the binary valued outcomes, the dice score for a perfect segmentation is one, the proposed method offers the best dice score along with best values for mean, medium and the maximal surface distance, and the Jaccard similarity compared to the other methods such as the dense $\mathrm{v}$ net and the watershed procedures. The figure. 4 below is the graphical representation of the overall percentage of performance metrics values of the proposed method and the previous state of art methods for the Jaccard similarity. Dice score, false negative and the false positive values.

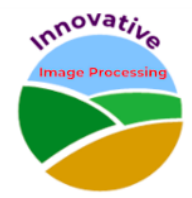


Journal of Innovative Image Processing (JIIP) (2020)

Vol.02/ No. 02

Pages: $110-120$

https://www.irojournals.com/iroiip/

DOI:_https://doi.org/10.36548/jiip.2020.2.006

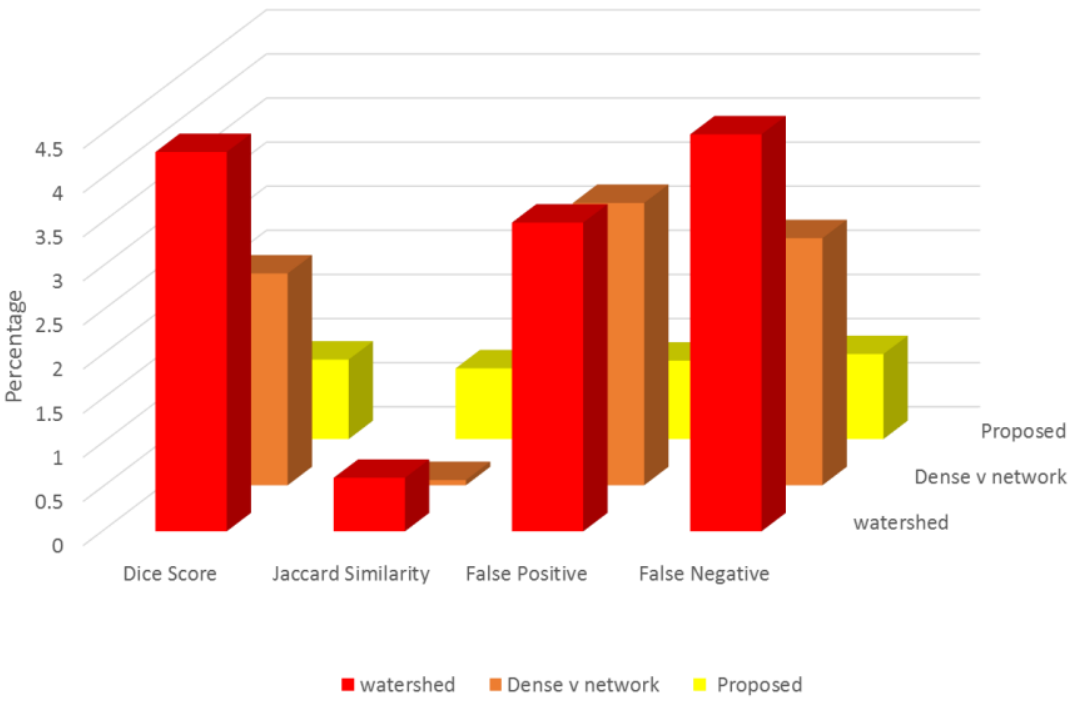

Figure.4 Graphical representation of Performance Metrics

The table below present the examination results of the proposed scheme on the basis of the dice score, for mean, medium, standard, and the maximal surface distance and the Jaccard similarity.

\begin{tabular}{|l|l|l|l|l|l|}
\hline $\begin{array}{l}\text { Performance } \\
\text { metrics }\end{array}$ & Data set 1 & Data set 2 & Data set 3 & Data set 4 & Data set 5 \\
\hline dice score & .901 & .923 & .911 & .914 & .917 \\
\hline $\begin{array}{l}\text { Jaccard } \\
\text { similarity }\end{array}$ & .82 & .83 & .82 & .84 & .81 \\
\hline $\begin{array}{l}\text { Mean } \\
\text { surface } \\
\text { distance }\end{array}$ & .025 & .027 & .034 & .043 & .022 \\
\hline $\begin{array}{l}\text { Median } \\
\text { surface } \\
\text { distance }\end{array}$ & .712 & .723 & .714 & .764 & .719 \\
\hline $\begin{array}{l}\text { Standard } \\
\text { surface } \\
\text { distance }\end{array}$ & .514 & .512 & .511 & .145 & .148 \\
\hline $\begin{array}{l}\text { Max surface } \\
\text { distance }\end{array}$ & 2.83 & 2.4 & 1 & 2.5 & 1.8 \\
\hline
\end{tabular}

Table.2 Results Acquired on the Proposed Scheme 
Journal of Innovative Image Processing (JIIP) (2020)

Vol.02/ No. 02

Pages: 110-120

https://www.irojournals.com/iroiip/

DOI:_https://doi.org/10.36548/jiip.2020.2.006

\section{Conclusion}

As the esophagus tumors are to be detected in its early stage, and the computed tomography images are very suitable to capture the changes even in the early developing stage, the paper uses the computed tomography images to resolve the issues identified due to the continuous variations in the position and the texture, intensity and as well as shape of the tumors causing complexities in developing an standard procedure that could be applied universally. The proposed method utilizes the active-contour based semiautomated segmentation along with the procedures of level set to segment the affected areas from the images of the esophagus acquired form the computed axial tomography. The strategy put forward segregates the work carried out into four major phases and achieves the results. This process is evaluated using the real life data sets based on the tumor occurrences in the different portions of the esophagus gathered from the cancer treatment hospital and evaluated on the basis of the performance metrics like dice similarity, mean, medium and the maximal surface distance, and the Jaccard similarity. The output score attained proves the efficiency of the proposed method compared to the other state of art methods.

\section{References}

[1] https://www.seattlecca.org/diseases/esophageal-cancer/esophageal-cancer-facts/esophagus

[2] Gibson, Eli, Francesco Giganti, Yipeng Hu, Ester Bonmati, Steve Bandula, Kurinchi Gurusamy, Brian Davidson, Stephen P. Pereira, Matthew J. Clarkson, and Dean C. Barratt. "Automatic multi-organ segmentation on abdominal CT with dense v-networks." IEEE transactions on medical imaging 37, no. 8 (2018): 1822-1834.

[3] Carvalho, L. E., Antonio Carlos Sobieranski, and Aldo von Wangenheim. "3D segmentation algorithms for computerized tomographic imaging: a systematic literature review." Journal of digital imaging 31, no. 6 (2018): 799-850.

[4] Koresh, Mr H. James Deva. "Quantization with Perception for Performance Improvement in HEVC for HDR Content." Journal of Innovative Image Processing (JIIP) 2, no. 01 (2020).

[5] Lenchik, Leon, Laura Heacock, Ashley A. Weaver, Robert D. Boutin, Tessa S. Cook, Jason Itri, Christopher G. Filippi et al. "Automated segmentation of tissues using CT and MRI: a systematic review." Academic radiology 26, no. 12 (2019): 1695-1706.

[6] Tam, Clara. "Machine Learning towards General Medical Image Segmentation." (2020).

[7] Horie, Miho. "Quantitative Imaging Using Computed Tomography for Evaluation of Chronic Lung Allograft Dysfunction." PhD diss., 2019.

[8] Manoharan, Samuel. "A Smart Image Processing Algorithm for Text Recognition Information Extraction and Vocalization for the Visually Challenged." Journal of Innovative Image Processing (JIIP) 1, no. 01 (2019): 31-38.

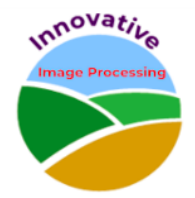


Journal of Innovative Image Processing (JIIP) (2020)

Vol.02/ No. 02

Pages: 110-120

https://www.irojournals.com/iroiip/

DOI:_https://doi.org/10.36548/jiip.2020.2.006

[9] Smith, Andrew. "Method for the detection and staging of liver fibrosis from image acquired data." U.S. Patent Application 16/196,158, filed March 21, 2019.

[10] Islam, Abm Rezbaul, Ali Alammari, and Bill Buckles. "Skin detection in image and video founded in clustering and region growing." In Mobile Multimedia/Image Processing, Security, and Applications 2019, vol. 10993, p. 109930V. International Society for Optics and Photonics, 2019.

[11] Bindhu, V. "Biomedical Image Analysis using Semantic Segmentation." Journal of Innovative Image Processing (JIIP) 1, no. 02 (2019): 91-101.

[12] Anshad, PY Muhammed, S. S. Kumar, and Shajeem Shahudheen. "Segmentation of chondroblastoma from medical images using modified region growing algorithm." Cluster Computing 22, no. 6 (2019): 13437-13444.

[13] Teng, Lin, Hang Li, Shoulin Yin, and Yang Sun. "Improved krill group-based region growing algorithm for image segmentation." International Journal of Image and Data Fusion 10, no. 4 (2019): 327-341.

[14] Anter, Ahmed M., and Aboul Ella Hassenian. "CT liver tumor segmentation hybrid approach using neutrosophic sets, fast fuzzy c-means and adaptive watershed algorithm." Artificial intelligence in medicine 97 (2019): 105-117.

[15] Song, Xiao, Gaoshan Deng, Yuanying Zhuang, and Nianyin Zeng. "An Improved Confidence Connected Liver Segmentation Method Based on Three Views of CT Images." IEEE Access 7 (2019): 58429-58434.

[16] Sathesh, A. "Performance Analysis of Granular Computing Model in Soft Computing Paradigm For Monitoring Of Fetal Echocardiography." Journal of Soft Computing Paradigm (JSCP) 1, no. 01 (2019): 14-23.

[17] Dias, Philipe Ambrozio, and Henry Medeiros. "Semantic segmentation refinement by Monte Carlo region growing of high confidence detections." In Asian Conference on Computer Vision, pp. 131-146. Springer, Cham, 2018.

[18] Nagesh, A. Sri, Dr GPS Varma, and Dr A. Govardhan. "An improved iterative watershed and morphological transformation techniques for segmentation of microarray images." IJCA Special Issue on "Computer Aided Soft Computing Techniques for Imaging and Biomedical Applications" CASCT 2 (2010): 77-87.

[19] Fourati, Wafa Abid, and Mohamed Salim Bouhlel. "Trabecular bone image segmentation using iterative watershed and multi resolution analysis." International Journal of Bio-Science and Bio-Technology 3, no. 2 (2011): 71-82.

[20] Masoumi, Hassan, Alireza Behrad, Mohammad Ali Pourmina, and Alireza Roosta. "Automatic liver segmentation in MRI images using an iterative watershed algorithm and artificial neural network." Biomedical signal processing and control 7, no. 5 (2012): 429-437.

[21] Yazdanpanah, Azadeh, Ghassan Hamarneh, Benjamin R. Smith, and Marinko V. Sarunic. "Segmentation of intra-retinal layers from optical coherence tomography images using an active contour approach." IEEE transactions on medical imaging 30, no. 2 (2010): 484-496.

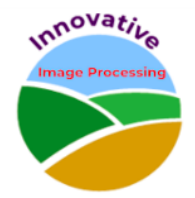


Journal of Innovative Image Processing (JIIP) (2020)

Vol.02/ No. 02

Pages: $110-120$

https://www.irojournals.com/iroiip/

DOI:_https://doi.org/10.36548/jiip.2020.2.006

[22] Karami, Ebrahim, Mohamed Shehata, Peter McGuire, and Andrew Smith. "A semi-automated technique for internal jugular vein segmentation in ultrasound images using active contours." In 2016 IEEE-EMBS International Conference on Biomedical and Health Informatics (BHI), pp. 184-187. IEEE, 2016.

\section{Authors Biography}

Dr. Bindhu V, is currently working as Professor and Head, in Department of Electronics and Communication Engineering, PPG Institute of Technology, Villankurichi Saravanampatti P.O, Coimbatore, Tamil Nadu, and India. Her major of research includes video communication, biomedical imaging, electronic imaging, image and video systems, and remote sensing.

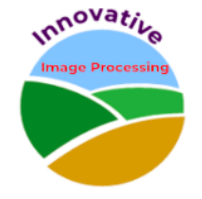

